\title{
Triggering of stroke by ambient temperature variation: A case-crossover study in Maputo, Mozambique
}

\author{
Joana Gomes ${ }^{\mathrm{a}, \mathrm{b}, *}$, Albertino Damasceno ${ }^{\mathrm{a}, \mathrm{c}, 2}$, Carla Carrilho ${ }^{\mathrm{c}, 2}$, Vitória Lobo ${ }^{\mathrm{c}, 2}$, \\ Hélder Lopes $^{c, 2}$, Tavares Madede ${ }^{c, 2}$, Pius Pravinrai ${ }^{c, 2}$, Carla Silva-Matos ${ }^{c, 2}$, \\ Domingos Diogo $^{c, 2}$, Ana Azevedo ${ }^{\mathrm{a}, \mathrm{b}, 1}$, Nuno Lunet ${ }^{\mathrm{a}, \mathrm{b}, 1}$
}

\footnotetext{
a Department of Clinical Epidemiology, Predictive Medicine and Public Health, University of Porto Medical School, Porto, Portugal

${ }^{\mathrm{b}}$ Institute of Public Health, University of Porto (ISPUP), Porto, Portugal

${ }^{\mathrm{c}}$ Faculty of Medicine, Eduardo Mondlane University, Maputo, Mozambique
}

\section{A R T I C L E I N F O}

\section{Article history:}

Received 3 August 2014

Received in revised form 8 December 2014

Accepted 9 December 2014

Available online 17 December 2014

\section{Keywords:}

Stroke

Ambient temperature

Trigger

Case-crossover

Mozambique

\begin{abstract}
A B S T R A C T
Objectives: The effect of ambient temperature as a stroke trigger is likely to differ by type of stroke and to depend on non-transient exposures that influence the risk of this outcome. We aimed to quantify the association between ambient temperature variation and stroke, according to clinical characteristics of the events, and other risk factors for stroke.

Methods: We conducted a case-crossover study based on a 1-year registry of the hospital admissions due to newly occurring ischemic and hemorrhagic stroke events in Maputo, Mozambique's capital city $(N=593)$. The case-period was defined as the 7 days before the stroke event, which was compared to two control periods (14-21 days and 21-28 days before the event). We computed humidity-and precipitationadjusted odds ratios (OR) and 95\% confidence intervals (95\%CI) using conditional logistic regression. Results: An association between minimum temperature declines higher than $2.4{ }^{\circ} \mathrm{C}$ in any two consecutive days in the previous week and the occurrence of stroke was observed only for first events $(\mathrm{OR}=1.43$, 95\%CI: 1.15-1.76). Stronger and statistically significant associations were observed for hemorrhagic stroke (OR = 1.50, 95\% CI: 1.07-2.09) and among subjects not exposed to risk factors, including smoking, high serum cholesterol or atrial fibrillation. No differences in the effect of temperature were found according to the patients' vital status 28 days after the event.

Conclusions: First stroke events, especially of the hemorrhagic type, were triggered by declines in the minimum temperature between consecutive days of the preceding week.
\end{abstract}

(c) 2014 Elsevier B.V. All rights reserved.

\section{Introduction}

Most stroke events are attributable to a relatively small number of well identified risk factors, corresponding to exposures that are essentially stable throughout time, which contribute to a higher background risk of stroke and to its overall burden [1,2]; however,

\footnotetext{
* Corresponding author at: Departamento de Epidemiologia Clínica, Medicina Preditiva e Saúde Pública, Faculdade de Medicina da Universidade do Porto, Al. Prof. Hernâni Monteiro, 4200-319 Porto, Portugal. Tel.: +351 225513652; fax: +351225513653

E-mail address: joanacostabgomes@hotmail.com (J. Gomes).

1 Address: Faculdade de Medicina da Universidade do Porto, Al. Prof. Hernâni Monteiro, 4200-319 Porto, Portugal.

2 Address: Hospital Central de Maputo, Avenida Eduardo Mondlane, Maputo, Mozambique.
}

these do not explain why an event occurs to a certain individual at a specific time $[3,4]$.

Transitory exposures, such as day-to-day variation in ambient temperature, have been shown to trigger the occurrence of stroke events [4-9], possibly by influencing, either directly or indirectly, biological factors that lead to an acute event (e.g., blood pressure and heart rate; serum concentration of lipids and vasoactive peptides; platelet aggregation) $[10,11]$. Nevertheless, the mechanisms underlying this triggering effect remain poorly understood, namely regarding the potential interaction with conventional risk factors for stroke and differences according to stroke subtype.

In an earlier study we showed that sudden decreases in the minimum temperatures were associated with an increase in the incidence rates of stroke in Maputo within 5-10 days [12]. In the present study we further addressed this topic by quantifying the association between ambient temperature variation 
on stroke occurrence, according to clinical characteristics of the events and other risk factors for stroke, using a case-cross over methodology.

\section{Patients and methods}

We conducted a case-crossover study based on a 12-month registry of the hospital admissions due to newly occurring stroke events in Maputo, the capital city of Mozambique.

\subsection{Identification of stroke events and data collection}

Between August 1, 2005 and July 31, 2006, we prospectively evaluated all patients admitted to any governmental or private hospital in Maputo (the Maputo Central Hospital, three general public hospitals, the Military Hospital, and six private clinics), living in town for $>12$ months, and suspected of having an incident stroke. The investigation followed the STEPS Stroke protocol, Step 1 (registration of hospitalized patients) according to the STEPS Stroke Manual Version 2.0 [13] as previously described [14].

Stroke was defined according to the World Health Organization clinical definition: "a focal (or at times global) neurological impairment of sudden onset, and lasting more than $24 \mathrm{~h}$ (or leading to death), and of presumed vascular origin [15]". During the study period trained interviewers were permanently at the Maputo Central Hospital Emergency Department for data collection. At other medical facilities a trained nurse in each shift was in charge of gathering information regarding patients with suspected stroke.

During the study period 651 stroke events were registered; CT scan or autopsy were performed in 601 (92.3\%), from which 351 (58.4\%) were classified as ischemic, $242(40.3 \%)$ as hemorrhagic and $8(1.3 \%)$ as subarachnoid hemorrhages. For this investigation, subarachnoid hemorrhages $(n=8)$ and stroke events with no classification regarding subtype $(n=50)$ were excluded leaving a total of 593 ischemic or hemorrhagic stroke events for data analysis. The date of symptom onset, as reported by the patient or a next-ofkin, was recorded and considered the day of the event (index date), regardless of the admission date.

Hypertension and diabetes were considered present when reported by the patients or their next of kin or when pharmacological treatment was prescribed on an inpatient or post-discharge basis. Information on socio-demographic characteristics, high serum cholesterol and current smoking was gathered using a standardized questionnaire, based on self-report. Atrial fibrillation was assessed through an electrocardiogram (EKG) performed to 416 patients at baseline or during the in-hospital stay. Glasgow score at admission was recorded and patients were followed up until the 28th day post-event (either through a pre-scheduled medical appointment or by telephone) to assess their vital status.

Data on the temperature in Maputo (maximum, minimum and mean temperatures for each day) were obtained from the Meteorological Institute of Mozambique, for the period between July 1, 2005 and July 31, 2006, along with mean relative daily humidity (\%) and total daily precipitation $\left(\mathrm{mm}^{3}\right)$ data.

\subsection{Case-crossover study design}

For each stroke event, a period of 7 days before the index date was defined as the case period, which was compared with two distinct event free periods, namely between day 14 and day 21 before the event (D14-D21) and between day 21 and day 28 before the event (D21-D28) (Fig. 1). To define the maximum daily variation in the minimum temperature in each 7-day case or control periods, we computed the difference between the minimum temperature in each day and the day before, and selected the figure corresponding to the highest decrease in any consecutive days. A 7-day case-period was selected because it corresponds to the midpoint of the 5-10-day period within which we previously observed a higher incidence of stroke after sudden temperature declines [12], and to facilitate the matching by day of the week, since it may also be associated with transitory exposures. Two control periods were selected to improve the statistical power of the study.

We computed the decrease in the minimum temperature between every two consecutive days, in the case period (D0-D7) and in each of the control periods (D14-D21 and D21-D28) [12], and the highest decrease in minimum temperature in each period was selected to define the exposure to temperature variation in case and control periods. In the present report we considered only the minimum temperature decreases as our previous analysis of incidence of stroke according to temperature in this setting identified the decline in minimum temperature as the most important trigger [12].

\subsection{Statistical analyses}

We computed odds ratios (OR) and 95\% confidence intervals $(95 \% \mathrm{CI})$, crude and adjusted for mean relative daily humidity (\%) and total daily precipitation $\left(\mathrm{mm}^{3}\right)$ in the case and control periods, as applicable, for the association between the decline in minimum temperature and stroke occurrence, using conditional logistic regression; the median of the distribution of the maximum dayto-day decrease in the minimum temperature in the case period was used as the cutoff to define the exposure to higher declines. Analyses were stratified by stroke subtype (first or recurrent and ischemic, hemorrhagic) age ( $\leq 44 ; 45-64$ or $\geq 65$ years), sex, and the presence of several stroke risk factors (hypertension, current smoking, high serum cholesterol, diabetes and atrial fibrillation), stroke severity (based on patients' Glasgow score at admission) and vital status assessed at the 28-day post-event (alive or dead). For stratified analyses the OR estimates were computed using all participants with available information for each variable.

All analyses were conducted using STATA, Version 11.0 (Stata Corporation, College Station, TX, USA).

\subsection{Ethics}

The study protocol was approved by the National Mozambican Ethics Committee and written informed consent was obtained for all participants.

\section{Results}

Nearly half the patients were women and aged between 45 and 64 years. A total of $86.2 \%$ of the patients had hypertension, 9.5\% were current smokers, $12.6 \%$ had high serum cholesterol and $13.4 \%$ had diabetes. Atrial fibrillation was present in just over $5 \%$ of the patients, mostly among those with ischemic stroke (Table 1).

During the 12-month study period, the median minimum temperatures were highest in February $\left(22.8^{\circ} \mathrm{C}\right)$ and lowest in June $\left(14.2^{\circ} \mathrm{C}\right)$. The monthly median of the highest decrease in minimum temperature between every 2 consecutive days in each 7-day period ranged from $1.3^{\circ} \mathrm{C}$ in July to $4.2^{\circ} \mathrm{C}$ in December (Fig. 2). When considering the cases among whom the highest decline in minimum temperature was higher for the case than for the control periods, the mean of the differences between each case period and the corresponding control periods D14-D21 and D21-D28 were $1.53^{\circ} \mathrm{C}$ and $1.42^{\circ} \mathrm{C}$, respectively.

Minimum temperature declines higher than $2.4^{\circ} \mathrm{C}$ in any consecutive days in the previous week were associated with the occurrence of stroke (crude $\mathrm{OR}=1.23,95 \% \mathrm{CI}$ : 1.02-1.49; adjusted $\mathrm{OR}=1.28,95 \% \mathrm{CI}$ : $1.05-1.56)$. However, no significant 


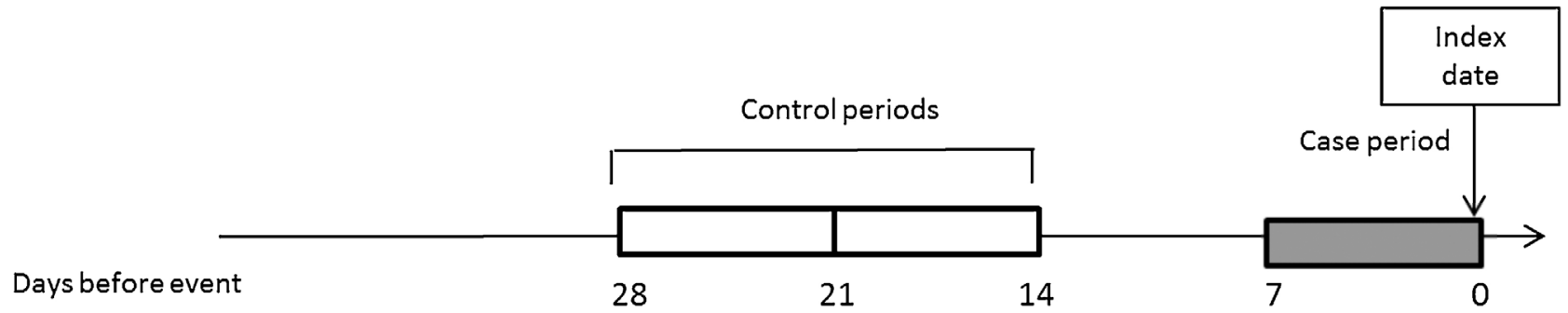

Fig. 1. Case (gray rectangle) and control-periods (white rectangles) used for the case-crossover analysis.

Table 1

Distribution of socio-demographic characteristics and risk factors for all patients and by first and recurrent events according to subtype.

\begin{tabular}{|c|c|c|c|c|c|}
\hline & \multirow[t]{2}{*}{ All events ${ }^{\mathrm{a}} n=593$} & \multicolumn{2}{|l|}{ First events ${ }^{\mathrm{a}}$} & \multicolumn{2}{|c|}{ Recurrent events ${ }^{\mathrm{a}}$} \\
\hline & & Ischemic $n=264$ & $\begin{array}{l}\text { Hemorrhagic } \\
\text { (intracerebral) } n=217\end{array}$ & Ischemic $n=87$ & $\begin{array}{l}\text { Hemorrhagic } \\
\text { (intracerebral) } n=25\end{array}$ \\
\hline \multicolumn{6}{|l|}{$\operatorname{Sex}[n(\%)]$} \\
\hline Women & $282(47.5)$ & $124(47.0)$ & $96(44.2)$ & $47(57.0)$ & $15(60.0)$ \\
\hline Men & $311(52.5)$ & $140(53.0)$ & $121(55.8)$ & $40(46.0)$ & $10(40.0)$ \\
\hline Age (years), [mean (SD)] & $58.8(13.1)$ & $60.5(13.8)$ & $54.7(11.5)$ & $63.9(11.8)$ & $57.2(11.8)$ \\
\hline \multicolumn{6}{|l|}{ Age (years), [n(\%)] } \\
\hline $25-44$ & $77(13.1)$ & $33(12.5)$ & $38(17.5)$ & $4(4.6)$ & $2(8.0)$ \\
\hline $45-64$ & $317(53.4)$ & $128(48.5)$ & $133(61.3)$ & $39(44.8)$ & $17(68.0)$ \\
\hline$\geq 65$ & $199(33.6)$ & $103(39.2)$ & $46(21.2)$ & $44(50.6)$ & $6(24.0)$ \\
\hline \multicolumn{6}{|l|}{ Risk factors, $[n(\%)]^{\mathrm{b}}$} \\
\hline Hypertension ${ }^{\mathrm{b}}$ & $513(86.5)$ & $213(80.7)$ & $196(90.3)$ & $80(92.0)$ & $24(96.0)$ \\
\hline Current smoking ${ }^{c}$ & $52(8.8)$ & $27(10.2)$ & $18(8.3)$ & $4(4.6)$ & $3(12.0)$ \\
\hline High serum cholesterol $^{\mathrm{d}}$ & $75(12.6)$ & $37(14.0)$ & $23(10.6)$ & $11(12.6)$ & $4(16.0)$ \\
\hline Diabetes $^{\mathrm{e}}$ & $79(13.3)$ & $40(15.2)$ & $20(9.2)$ & $16(18.4)$ & $3(12.0)$ \\
\hline Atrial fibrillation ${ }^{\mathrm{f}}$ & $33(5.6)$ & $16(6.1)$ & $3(1.4)$ & $13(14.9)$ & $1(4.0)$ \\
\hline
\end{tabular}

SD, standard deviation.

a Including 593 cases with available information regarding the stroke subtype and after the exclusion of eight subarachnoid hemorrhages.

b Data are missing from 36 subjects.

c Data are missing from 17 subjects.

d Data are missing from 77 subjects.

e Data are missing from 34 subjects.

f Data are missing from 32 subjects.

effect was observed for recurrent events (adjusted OR $=0.96,95 \% \mathrm{CI}$ : $0.61-1.50)$ and an increased risk was observed only for first events (adjusted $\mathrm{OR}=1.39,95 \% \mathrm{CI}$ : 1.11-1.74). Among the latter (Table 2), there were no meaningful differences between men and women, but stronger and statistically significant associations were observed for hemorrhagic stroke (adjusted $\mathrm{OR}=1.50,95 \% \mathrm{CI}$ : 1.07-2.09), hypertensive individuals (adjusted $\mathrm{OR}=1.37,95 \% \mathrm{CI}$ : $1.08-1.75$ ), non-smokers (adjusted $\mathrm{OR}=1.42,95 \% \mathrm{CI}$ : $1.12-1.80$ ) and subjects with lower serum cholesterol levels (adjusted $\mathrm{OR}=1.53,95 \% \mathrm{CI}$ : $1.20-1.96)$. No differences were found regarding stroke severity or vital status 28 days after the event (Table 2 ).

For patients arriving to the hospital less than $24 \mathrm{~h}$ after stroke onset adjusted OR was 1.24 (95\%Cl: $0.92-1.67)$ and for those arriving to the hospital more than $24 \mathrm{~h}$ after stroke onset adjusted OR was 1.62 (95\%CI: 1.15-2.27). Considering the difference between the day before the event and the index date as the case period and to corresponding control periods (D8-D7 and D15-D14), the overall adjusted $\mathrm{OR}$ for decreases higher than $2.4{ }^{\circ} \mathrm{C}$ was 1.06 (95\% CI: $0.82-1.37)$.

\section{Discussion}

This study confirms that stroke events may be triggered by drops in the minimum temperature, in a setting with a tropical, high humidity climate. It adds to our previous findings evidence that this association only applies to first events, mainly hemorrhagic, and that there was no relation with the 28-day vital status of these patients.
Most previous studies aiming to evaluate the relationship between temperature and stroke occurrence found stroke to be associated with lower temperatures [16-19]. A large study conducted in 17 countries, including three from Africa (Kenya, Zambia and Zimbabwe) showed that hospitalizations due to stroke were associated with lower environmental air temperature and that this effect was verified for lags between drops in temperature and stroke occurrence not larger than 1 month [20], which is compatible with our data. The case-crossover methodology allows for an efficient control of time-invariant confounders, such as socioeconomic status and other socio-demographic characteristics and factors associated with the day of the week (e.g., patterns of predominant alcohol drinking in the weekends), contributing to the validity of the risk estimates $[21,22]$. The fact that the control periods precede the case period only by $2-3$ weeks also accounts for a potential effect of seasonal variation.

However, to our knowledge only a few studies have shown the association between ambient temperature and stroke occurrence using this study design $[6,7,23]$. One investigation conducted in South Korea showed that stroke risk was associated with decreases in ambient temperature of $5^{\circ} \mathrm{C}$ for a risk period of 1 day (adjusted $\mathrm{OR}=1.4,95 \% \mathrm{CI}: 1.1-1.6$ ) [6]. A study conducted in Germany found no relevant associations between temperature and stroke although there was a tendency for higher risk with changes in maximum temperature (either downwards or upwards) greater than $5^{\circ} \mathrm{C}$ (one day lag-time); however, the authors also analyzed minimum and mean temperatures obtaining similar results [7]. More recently, a case-crossover conducted in The United States showed that the risk 


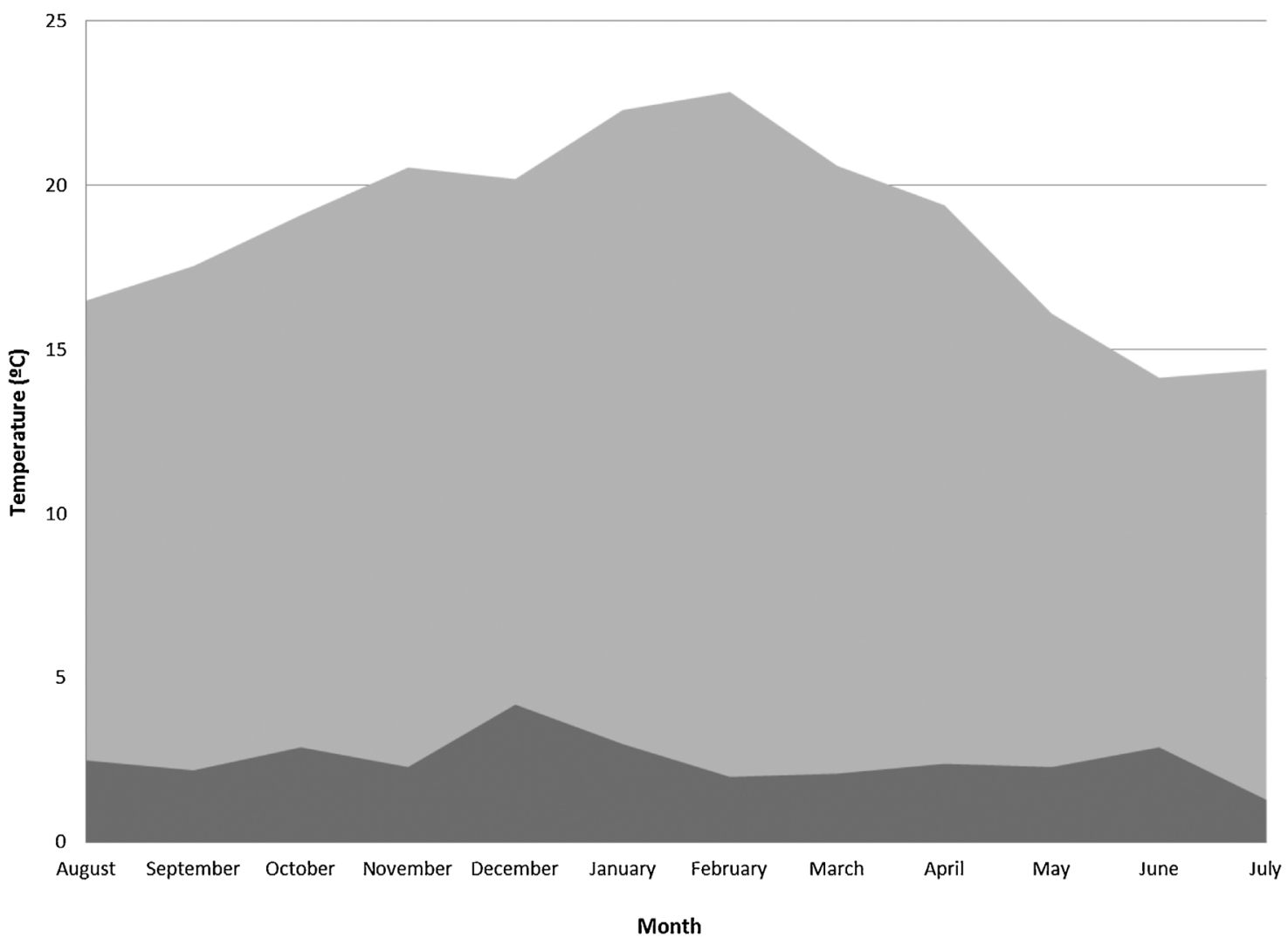

Minimum temperature (monthly median)

Maximum decline in minimum temperature between two consecutive days,

computed for 7-day periods (monthly median)

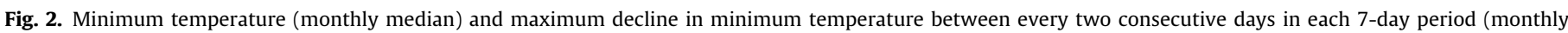
median), between August 1, 2005 and July 31, 2006.

of ischemic stroke was higher following a $5^{\circ} \mathrm{C}$ decrement in average apparent temperature over the 2 days preceding symptom onset. The risk did not vary across strata of stroke risk factors namely sex, age ( $<65 v s$. $\geq 65$ ), smoking history (current, former, never), history of diabetes mellitus, hypertension or atrial fibrillation; this study, however, did not include hemorrhagic stroke events and was conducted in a population with different baseline characteristics (mostly white, younger patients, with lower prevalence of hypertension) [23].

The different lag-times and magnitude of temperature variation used in the previous studies may reflect the climatic differences between countries, since Mozambique is a country with fairly high temperatures throughout the year and small day-to-day variations, which may have a greater impact on the biological mechanisms involved due to a more difficult adaptation [24,25]. Also, as our findings show a stronger association with hemorrhagic stroke events, the effect of ambient temperature in settings with lower proportion of this subtype could be less pronounced. These heterogeneous findings reinforce the need for locale-specific data for further understanding the effects of temperature on the occurrence of stroke. It was previously shown that mean systolic and diastolic blood pressure tend to be higher during colder months and that cold exposure can exacerbate hypertension in hypertensive individuals [10]; this may reflect increasing systemic vascular resistance and oxygen demand, potentially leading to ischemia, as well as the fact that individuals with hypertension are less effective in cold adaptation due to autonomic nervous system dysregulation [26]. The latter may also apply to diabetic subjects [27], but there was no significantly higher risk in these patients.

The fact that the effect of temperature on stroke occurrence was not immediate and was verified for a relatively small temperature drop when compared to previous evidence [28], supports the theory that although hypertension is likely to be involved in the relationship between ambient temperature and stroke, the mechanisms underlining this association go further than the instant influence on blood pressure levels and could be mediated by the pro-inflammatory state and hypertension-associated dysautonomia or to be influenced by other variables such as infections, changes in vasoactive peptides serum concentration, hypercoagulability and others [10,29]. Further clarification of the mechanisms through which temperature can elicit stroke on an hypertensive individual is necessary.

We found no association with temperature drops for recurrent stroke events. Previously conducted investigation showed that recurrent stroke risk varied according to geographical region, independently of the presence of stroke risk factors [30]. Though not fully understood, differences might be related to factors such as genetic variation, environmental factors, healthcare accessibility and infectious disease exposure, which can also explain our results.

Most previous studies showed higher susceptibility to temperature changes in the elderly, due to less effective cold adaptation [6,31]. In our study, no significant variation was observed across age-groups, which may reflect the specificities of this setting regarding the mean and day-to-day variation in temperature, as 
Table 2

Association between the maximum decrease in minimum temperature and incidence of a first stroke event, using 0-7 days before the events as case period (D0-D7) and 14-21 and 21-28 days before the event as the control periods (D14-D21 and D21-D28, respectively) $(N=593)$.

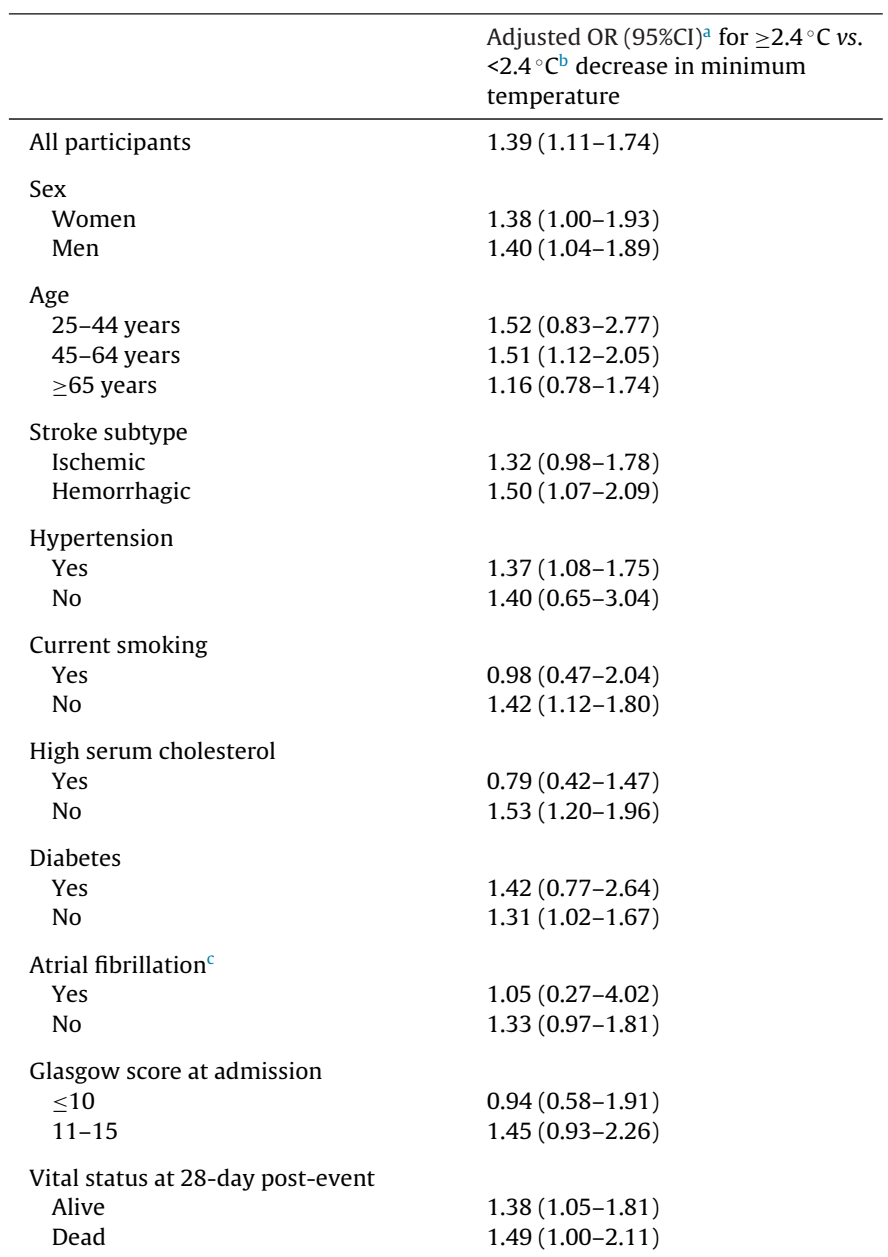

a OR adjusted for mean relative daily humidity (\%) and mean precipitation $\left(\mathrm{mm}^{3}\right)$ in each case/control period.

b $2.4{ }^{\circ} \mathrm{C}$ corresponds to the median value of the distribution of the maximum dayto-day decrease in the minimum temperature in the case period.

c Analysis conducted only for ischemic events.

well as more difficulties in the access to health care services by older people.

The present study provides relevant information for a better understanding of the triggering of stroke by sudden temperature changes, from a setting where research on this topic is scarce, and using a robust study design. There are, however, some limitations that need to be addressed. This unidirectional case-crossover analysis does not allow for controlling of time-variant confounders (e.g.: level of air pollutants; adherence to antihypertensive therapy) that could not be taken into account. Previous investigation show that higher pollution levels are associated with an increased risk of stroke [32], and it would be interesting to study its influence in the Mozambican context; nevertheless, Maputo does not have heavy industry and road traffic has increased mostly in the more recent years. Our results are also limited by the fact that the definition of the day of the symptoms onset may not be accurate, as many patients arrived in the hospital several days after the index date, thus increasing the possibility of memory bias and consequently, the misclassification of the case-period [14]. Although we may hypothesize that bad weather may contribute to a more difficult access to health services, misclassification may be less pronounced when considering the day-to-day variation in the minimum temperature across a 7-day period. Despite the exposure to ambient temperature is influenced by housing conditions, type of work and leisure activities, in the Mozambican context, where most of the population lives in houses with poor thermal insulation, an high correlation between indoor and outdoor temperatures to which most of the participants were exposed may be expected [33].

Since no previous registries were conducted in Mozambique including also patients not seeking health care due to stroke, it is difficult to predict the extent to which this sample underestimates stroke incidence in Maputo during this period, and therefore our conclusions apply only to cases of hospitalized stroke patients. Meteorological conditions may have contributed for some patients not having reached an hospital, and therefore, further studies are needed to understand the effect of temperature on stroke events that were taken care in the community.

Because we had no information regarding traveling in the period before stroke, or in the corresponding control periods, the interpretation of our results requires the assumption that traveling for regions with different patterns of temperature changes is unlikely to have occurred.

The fact that some patients arrived to the hospital several days after stroke increases the possibility of recall bias, regarding the day of symptom onset. We have conducted a sensitivity analysis by stratifying patients according to delay on reaching the hospital ( $<1$ day $v s . \geq 1$ day) and results there was a slighter higher risk of stroke for patients arriving to the hospital more than 1 day after stroke symptom onset. This result probably also reflects differences between these groups regarding stroke severity and access to medical care.

Misclassification regarding risk factors assessment could also be present, although only the assessment of smoking status and high serum cholesterol were based exclusively on self-report. Regarding atrial fibrillation, under diagnoses is possible as results were based on a single EKG. Nevertheless, African-origin stroke patients have lower prevalence of atrial fibrillation and previous evidence has shown an atrial fibrillation prevalence of $6.8 \%$ in black patients, which is not far from the prevalence found in our study which only accounts for stroke patients [34]. No statistically significant results were observed when testing formally for the presence of effect modification (data not shown), most likely due to the small sample in many of the strata analyzed; further studies with larger samples are needed to confirm our findings.

\section{Conclusions}

This study showed the impact of temperature changes in the triggering of stroke events, in Maputo. Although the triggering effect observed was similar regardless of the vital status of the patients 28 days after the event, these results may be useful for the planning of health-care services in Maputo, namely by ensuring that the resources needed for the diagnosis and acute management of stroke are available in these periods, which is relevant in a setting here health resources are scarce. Future research should continue to pursue the clarification of the mechanisms underlying the cascade of events that underlie the relation between temperature changes and the triggering of stroke.

\section{Sources of funding}

Data collection funded by the Mozambican Ministry of Health and the African Regional Office of the World Health Organization. The work of A.D. and C.C. was supported by the Grant Number R24TW008908 from the Fogarty International Center. The content is solely the responsibility of the authors and does not necessarily 
represent the official views of the Fogarty International Center or the National Institutes of Health. This award is supported by funds provided to the NIH and HRSA under the "Tom Lantos and Henry Hyde United States Leadership Against HIV/AIDS, Tuberculosis, and Malaria Reauthorization Act of 2008," Public Law 110-293, which is more commonly known as the U.S. Presidents Emergency Plan for AIDS Relief (PEPFAR). Co funding is also provided by the NIH Office of Research on Women's Health and the Office of AIDS Research.

\section{Conflicts of interest}

None.

\section{References}

[1] Rundek T, Sacco RL. Risk factor management to prevent first stroke. Neurol Clin 2008;26:1007-45.

[2] Straus SE, Majumdar SR, McAlister FA. New evidence for stroke prevention: scientific review. JAMA 2002;288:1388-95.

[3] Guiraud V, Amor MB, Mas JL, Touze E. Triggers of ischemic stroke: a systematic review. Stroke 2010;41:2669-77.

[4] Tofler GH, Muller JE. Triggering of acute cardiovascular disease and potential preventive strategies. Circulation 2006;114:1863-72.

[5] Coelho FM, Santos BF, Cendoroglo Neto M, Lisboa LF, Cypriano AS, Lopes TO, et al. Temperature variation in the 24 hours before the initial symptoms of stroke. Arq Neuropsiquiatr 2010;68:242-5.

[6] Hong YC, Rha JH, Lee JT, Ha EH, Kwon HJ, Kim H. Ischemic stroke associated with decrease in temperature. Epidemiology 2003;14:473-8.

[7] Kyobutungi C, Grau A, Stieglbauer G, Becher H. Absolute temperature, temperature changes and stroke risk: a case-crossover study. Eur J Epidemiol 2005;20:693-8.

[8] Elkind MS. Why now? Moving from stroke risk factors to stroke triggers. Curr Opin Neurol 2007:20:51-7.

[9] Jimenez-Conde J, Ois A, Gomis M, Rodriguez-Campello A, Cuadrado-Godia E, Subirana I, et al. Weather as a trigger of stroke. Daily meteorological factors and incidence of stroke subtypes. Cerebrovasc Dis 2008;26:348-54

[10] Cheng X, Su H. Effects of climatic temperature stress on cardiovascular diseases, Eur J Intern Med 2010;21:164-7.

[11] Manfredini R, Gallerani M, Portaluppi F, Salmi R, Fersini C. Chronobiological patterns of onset of acute cerebrovascular diseases. Thromb Res 1997:88: 451-63.

[12] Gomes J, Damasceno A, Carrilho C, Lobo V, Lopes H, Madede T, et al. The effect of season and temperature variation on hospital admissions for incident stroke events in Maputo, Mozambique. J Stroke Cerebrovasc Dis 2014:23:271-7.

[13] World Health Organization. WHO STEPS stroke manual: the WHO STEPwise approach to stroke surveillance. Geneva: World Health Organization; 2006. Available at: http://www.who.int/chp/steps/Manual.pdf [accessed March, 2014].

[14] Damasceno A, Gomes J, Azevedo A, Carrilho C, Lobo V, Lopes H, et al. An epidemiological study of stroke hospitalizations in Maputo, Mozambique: a high burden of disease in a resource-poor country. Stroke 2010:41:2463-9.
[15] Hatano S. Experience from a multicentre stroke register: a preliminary report. Bull World Health Organ 1976;54:541-53.

[16] Chen ZY, Chang SF, Su CL. Weather and stroke in a subtropical area: Ilan, Taiwan. Stroke 1995:26:569-72.

[17] Jakovljevic D, Salomaa V, Sivenius J, Tamminen M, Sarti C, Salmi K, et al. Seasonal variation in the occurrence of stroke in a Finnish adult population. The FINMONICA stroke register. Finnish monitoring trends and determinants in cardiovascular disease. Stroke 1996;27:1774-9.

[18] Oberg AL, Ferguson JA, McIntyre LM, Horner RD. Incidence of stroke and season of the year: evidence of an association. Am J Epidemiol 2000;152:558-64.

[19] Azevedo E, Ribeiro JA, Lopes F, Martins R, Barros H. Cold: a risk factor for stroke. J Neurol 1995;242:217-21.

[20] Chang CL, Shipley M, Marmot M, Poulter N. Lower ambient temperature was associated with an increased risk of hospitalization for stroke and acute myocardial infarction in young women. J Clin Epidemiol 2004;57:749-57.

[21] Padrao P, Damasceno A, Silva-Matos C, Laszczynska O, Prista A, Gouveia L, et al, Alcohol consumption in Mozambique: regular consumption, weekly pattern and binge drinking. Drug Alcohol Depend 2011:115:87-93.

[22] Bateson TF, Schwartz J. Control for seasonal variation and time trend in casecrossover studies of acute effects of environmental exposures. Epidemiology 1999;10:539-44.

[23] Mostofsky E, Wilker E, Schwartz J, Zanobetti A, Gold D, Wellenius G, et al. Shortterm changes in ambient temperature and risk of ischemic stroke. Cerebrovasc Dis Extra 2014:9-18.

[24] Analitis A, Katsouyanni K, Biggeri A, Baccini M, Forsberg B, Bisanti L, etal. Effects of cold weather on mortality: results from 15 European cities within the PHEWE project. Am J Epidemiol 2008;168:1397-408.

[25] Conlon KC, Rajkovich NB, White-Newsome JL, Larsen L, O'Neill MS. Preventing cold-related morbidity and mortality in a changing climate. Maturitas 2011;69:197-202.

[26] Abboud FM, Harwani SC, Chapleau MW. Autonomic neural regulation of the immune system: implications for hypertension and cardiovascular disease. Hypertension 2012;59:755-62.

[27] Scott AR, MacDonald IA, Bennett T, Tattersall RB. Abnormal thermoregulation in diabetic autonomic neuropathy. Diabetes 1988;37:961-8.

[28] Brennan PJ, Greenberg G, Miall WE, Thompson SG. Seasonal variation in arterial blood pressure. Br Med J (Clin Res Ed) 1982;285:919-23.

[29] Zurru MC, Alonzo C, Brescacin L, Romano M, Camera LA, Waisman G, et al. Recent respiratory infection predicts atherothrombotic stroke: case-control study in a Buenos Aires healthcare system. Stroke 2009;40:1986-90.

[30] Allen NB, Holford TR, Bracken MB, Goldstein LB, Howard G, Wang Y, et al. Geographic variation in one-year recurrent ischemic stroke rates for elderly Medicare beneficiaries in the USA. Neuroepidemiology 2010;34:123-9.

[31] Wang XY, Barnett AG, Hu W, Tong S. Temperature variation and emergency hospital admissions for stroke in Brisbane, Australia, 1996-2005. Int J Biometeoro 2009;53:535-41.

[32] Corea F, Silvestrelli G, Baccarelli A, Giua A, Previdi P, Siliprandi G, et al. Airborne pollutants and lacunar stroke: a case cross-over analysis on stroke unit admissions. Neurol Int 2012;4:e11.

[33] Schluter PJ, Macey PM, Ford RP. The relationship between inside and outside ambient temperatures in Christchurch, New Zealand. Paediatr Perinat Epidemiol 2000;14:275-82.

[34] Hajat C, Dundas R, Stewart JA, Lawrence F, Rudd AG, Howard R, et al Cerebrovascular risk factors and stroke subtypes: differences between ethnic groups. Stroke 2001;32:37-42. 